\title{
CERTIFICACIÓN PROFESIONAL COMO HERRAMIENTA FORMATIVA EN GRADO EN INGENIERÍA INFORMÁTICA EN LA ESCUELA POLITÉCNICA SUPERIOR DE CÓRDOBA
}

\author{
PROFESSIONAL CERTIFICATION AS A TEACHING TOOL IN COMPUTER SCIENCE \\ DEGREE AT THE HIGHER POLYTECHNIC SCHOOL OF CORDOBA
}

\author{
J. M. Palomares Muñoz ${ }^{1}$, J. C. Gámez Granados, J. Gómez Luna, J. M. Soto Hidalgo, A. Zafra Gómez \\ Universidad de Córdoba (España)
}

\begin{abstract}
RESUMEN: Existen múltiples empresas que emiten certificaciones de competencias profesionales dentro del ámbito de las Ingenierías. Estas certificaciones están siendo muy demandadas por las empresas empleadoras ya que permiten demostrar la cualificación de los solicitantes desde un punto de vista teórico y práctico muy exigente. Esto ha llevado a que muchos alumnos y egresados hayan tomado cursos de preparación para los exámenes de certificación. Una de las más relevantes es CISCO, que realiza certificaciones de competencias de Redes de Computadores. Un grupo de profesores de la EPSC de la Universidad de Córdoba ha planteado adaptar los contenidos de diversas asignaturas de la Titulación Oficial de Grado en Ingeniería Informática para que el alumnado cubra todos los requisitos formativos (tanto teóricos como prácticos) exigidos por la empresa CISCO para las diversas certificaciones en materia de Redes de Comunicación entre Ordenadores. Además, esta adaptación debe hacerse sin alterar el documento Verifica, aprobado por la Agencia Nacional de Acreditación y Evaluación (ANECA).
\end{abstract}

Palabras clave: Certificación Profesional, Ingeniería

ABSTRACT: There are a lot of enterprises which provide certifications of knowledge and skills in professional competences within the Engineering field. These certifications are highly demanded by employers, because they demonstrate the qualification of the applicants from a very demanding theoretical and practical point of view. Thus, a lot of students and graduates have enrolled in training courses for the certification exams. One of the most important corporations is $\mathrm{CISCO}$, which provides certifications in the field of Computer Networking. A group of professors from the Higher Polytechnic School of Cordoba from the University of Cordoba (Spain) has adapted the contents of several subjects of the Computer Science Degree, in order to cover all the educational requirements required by $\mathrm{CISCO}$ for the Computer Networking field. Besides, this adaptation has to be taken without any modification in the VERIFICA document, approved by the Spanish National Certification and Evaluation Agency (ANECA).

Key words: Professional Certification, Engineering

\section{Introducción}

La certificación de competencias profesionales en el ámbito de las Ingenierías está presente tanto en el plano académico como en el empresarial desde hace mucho tiempo. Con estas

\footnotetext{
${ }^{1}$ Dirección de contacto: Dpto. Arquitectura de Computadores, Electrónica y Tecnología Electrónica. Escuela Politécnica Superior de Córdoba. Universidad de Córdoba, España. Edificio Leonardo da Vinci - Campus Universitario de Rabanales 14071- Córdoba (España).jmpalomares@uco.es
} 
certificaciones se pretende garantizar que los solicitantes poseen conocimientos y competencias de un determinado ámbito del conocimiento. En muchos países, como por ejemplo España, los Colegios Profesionales son responsables de certificar las competencias profesionales de los solicitantes para todas aquellas profesiones reguladas. El mecanismo más común para asegurar que los solicitantes poseen las competencias profesionales es mediante la presentación de Títulos Universitarios oficiales asociados a las diversas profesiones reguladas. El problema aparece en nuevas profesiones que no están reguladas por ley, y por tanto, o no existen Colegios Profesionales Oficiales, o no están reconocidos a nivel oficial.

Por otra parte, en muchos países, en los que no existen Colegios Profesionales, son las diversas empresas del ámbito de la profesión las que evalúan a los solicitantes bajo unos determinados requisitos, que las propias empresas exigen. Los solicitantes que superen las pruebas obtienen unos certificados emitidos por la empresa evaluadora. ¿Qué validez tienen estos certificados? Dichos certificados tienen la validez que el resto de empresas y empleadores quieran darle. Esto está sujeto a la fiabilidad que dicha empresa tenga en el ámbito profesional y la credibilidad externa que muestre la línea de certificación de dicha empresa. Es por ello, que casi todas las grandes empresas mundiales que trabajan en el ámbito de las Ingenierías suelen tener dentro de sus líneas de negocio, la certificación profesional en el campo de trabajo de dichas empresas.

Esta opción ha tomado mucha fuerza y reconocimiento a nivel mundial, llegándose a exigir en muchas ofertas de trabajo antes que las titulaciones oficiales. Una pregunta que se debería hacer la universidad en general, es porqué esto está ocurriendo. Es probable que determinados conocimientos y competencias no estén suficientemente actualizados y debido a la rigidez de los planes de estudio, las empresas prefieran el dinamismo de estas certificaciones que se adaptan con mayor rapidez a cualquier novedad que aparezca en cada campo científico-técnico.

Integrar las novedades y las nuevas competencias que surgen en la progresión innovadora de las ingenierías en los títulos oficiales puede conllevar la necesidad de modificar los contenidos de los títulos. En España, alterar un plan de estudios de una titulación oficial requiere un esfuerzo considerable, ya que exige modificar el documento VERIFICA, que previamente ha debido ser acreditado por la ANECA para su implantación como título oficial. Por ello, los centros y universidades son reticentes a cualquier tipo de modificación sustancial de los planes de estudio acreditados. Por tanto, hay nuevas competencias y contenidos que no se integran en los títulos de manera inmediata, sino hasta que surge la obligación de presentar un nuevo título oficial, lo que puede hacer que transcurran bastantes años hasta su incorporación en los temarios. Esto provoca un desfase temporal que puede ir en contra de los estudiantes, que a pesar de tener un título universitario oficial, se encuentren en desventaja frente a otros estudiantes que hayan obtenido la certificación de las competencias profesionales a través de estos otros métodos.

El presente artículo se ha estructurado como sigue. En la Sección 1 se describen brevemente los modelos formativos europeos que permiten la adquisición de competencias profesionales. La Sección 2 indica cómo está la situación de las certificaciones profesionales en España. La Sección 3 se centra en particular en las competencias profesionales en el ámbito de las Redes de Computadores dentro de los títulos oficiales de Informática en España. Tras ello, en la Sección 4 se expone y describe el certificado CCNA propuesto por la empresa Cisco System, de gran aceptación mundial en el campo de las Redes de Computadores. La adaptación de las asignaturas del Grado en Ingeniería Informática de la Universidad de Córdoba a lo exigido por la certificación CCNA de Cisco System se describe en la Sección 5. La Sección 6 muestra el resultado de la aplicación de esta propuesta de adaptación de los contenidos del título oficial con las recomendaciones de la certificación CCNA.Tras ello, se presenta la sección de Conclusiones, 
en la que se muestran los principales logros de esta experiencia y su posible uso en otros ámbitos. Finalmente, se añade una sección de agradecimientos y las referencias bibliográficas.

\section{Modelos formativos para la adquisición de competencias profesionales}

Haciendo una breve recopilación de los modelos formativos oficiales (con reconocimiento estatal) en Europa, se pueden identificar tres modelos principales de formación profesional (Merle, 1997), que pretenden otorgar a los estudiantes competencias y conocimientos de cada determinado ámbito profesional. El primer modelo, denominado modelo alemán (o modelo de formación dual), integra competencias prácticas adquiridas directamente en las empresas con conocimientos teóricos adquiridos en el aula. Las competencias se ven muy influenciados por las empresas, que ofertan periodos de prácticas en ellas y que son los que definen qué empleos deben tenerse en consideración. En el modelo inglés, el gobierno británico ha definido una serie de competencias profesionales libremente validables para cada profesión, que junto con una red de organismos de validación (independientes parcialmente de las empresas contratantes y de los entes formativos que imparten los estudios) permiten certificar los conocimientos de los estudiantes. Finalmente, el modelo de "Libros Blancos" ha sido el que se ha impuesto en un mayor número de países europeos. Este modelo, también llamado de "acreditación de competencias", muestra algunas similitudes con el modelo inglés aunque se separa de éste al no unirse a ninguna profesión concreta. Es decir, se acredita el dominio de un conjunto de competencias profesionales, sin tener en cuenta ninguna profesión en particular.

Estos tres modelos están orientados hacia los estudios de tercer grado no universitarios (Formación Profesional, Ciclos Formativos de grado superior). Sin embargo, son de plena aplicación en el ámbito universitario para la obtención de titulaciones que tengan reconocimiento de competencias profesionales.

Otra alternativa es la formación especializada no oficial. Al no estar sujeta a las restricciones impuestas por las diversas normas y reglamentos públicos, que suelen ser más rígidos, permiten modificaciones más dinámicas de sus contenidos y competencias, adaptándose con mayor celeridad a los cambios que la tecnología suele traer. Es evidente, que este dinamismo tiene su contrapartida, se pueden incorporar tecnologías que caigan en desuso rápidamente, que se vean superadas por otras o que sean propietarias de una determinada empresa y no sean de uso generalizado. De manera adicional, el hecho de que no estén reguladas por un ente público no significa que no tengan una regulación común. Habitualmente, son las propias certificadoras las que emiten sus propias normas para garantizar que se incorporan (o se retiran) de los títulos, temarios y contenidos de las certificaciones todos aquellos aspectos relevantes (o que hayan dejado de serlo, respectivamente). Esto se realiza habitualmente mediante comités científicos conformados por expertos internacionales de reconocido prestigio de distintos ámbitos: empresas, universidades, centros de investigación, etc. Estos comités deben trabajar por garantizar tanto la adaptación a las nuevas tecnologías emergentes como la adhesión a conocimientos establecidos que deben asegurarse como competencias específicas de dicha materia. Gracias a estos comités y a la competencia entre las diferentes entidades certificadoras, se logra que, de facto, se acepten a nivel mundial aquellas certificaciones no oficiales que han alcanzado un gran nivel de relevancia.

Por tanto, actualmente conviven dos grandes mecanismos certificadores: los adquiridos por haber cursado y aprobado títulos oficiales y los obtenidos mediante exámenes de certificación de títulos no oficiales. La gran pregunta que surge es: ¿compiten entre sí ambos mecanismos 0 es posible una sinergia entre ambos? 


\section{Certificaciones profesionales en el ámbito de la Informática en España}

La Informática es una ingeniería, que hasta el momento, no posee competencias profesionales reguladas ni limitación en el ejercicio de la profesión ni en España ni en ningún otro país europeo. En cualquier caso, la Informática se ha consolidado como una profesión ya que presenta (ANECA, 2005):

- Un campo duradero de preocupación humana.

- Un cuerpo codificado de principios (conocimiento conceptual).

- Un cuerpo codificado de prácticas (conocimiento experimental, incluyendo competencia).

- Estándares de competencia ética y práctica.

Por tanto, al ser unos estudios que no conducen al ejercicio de una profesión regulada, los estudios oficiales no proporcionan competencias profesionales. Ya que en España existen Colegios Profesionales de Informática de ámbito regional, que están unificados bajo los ConsejosGenerales de ámbito nacional, sería factible que éstas proporcionaran el marco de certificación profesional, aunque dichas certificaciones no tuviesen validez regulatoria oficial. Sin embargo, los colegios profesionales de Informática no tienen dentro de sus funciones reconocidas por la ley, la de la emisión de certificación profesional (Decreto 517/2015, 2015); (Decreto 518/2015, 2015).

Los estudios universitarios oficiales de Grado en Ingeniería Informática se han diseñado bajo el modelo de "Libros Blancos" (ANECA, 2005), incluyéndose un conjunto de competencias básicas, competencias específicas comunes y competencias de tecnología específica. Dentro de estas últimas, se encuentran las competencias propias de Ingeniería Informática y, en el caso que de fuese una profesión regulada, entre ellas estarían las competencias profesionales. Por tanto, el cursar el Grado en Ingeniería Informática proporciona a los estudiantes un conjunto de conocimientos y competencias específicas de la tecnología informática. Sin embargo, ¿son suficientes esas competencias para las empresas?

En gran medida, parece que sí, según muestran los requisitos de los empleos de los principales portales (ticjob.es, 2015); (infojobs.net, 2015) de empleos ligados a Informática de España, ya que la gran mayoría de ofertas de trabajo relativos a Informática únicamente solicitan un título universitario de Informática (Ingeniería Técnica, Grado, Diplomatura o superior).Sin embargo, si se observa con detenimiento las ofertas se puede observar que dichas ofertas de empleo son en pequeñas y medianas empresas (PYME). Por el contrario, en grandes empresas y corporaciones, y sobre todo para puestos de trabajo de categoría superior (analista senior 0 superior) sí que requieren, además del título universitario, determinados certificados profesionales. Por lo tanto, se puede deducir que las certificaciones profesionalesprivadas (en oposición a las emitidas por organismos públicos, universidades, colegios profesionales, etc.) se valoran muy positivamente por las empresas, sobre todo para puestos de trabajo de alta cualificación.

\subsection{Principales Certificaciones Profesionales Privadas en el ámbito de la Informática}

Existe un volumen elevadísimo de certificaciones profesionales privadasa nivel mundial, con variados contenidos y requisitos (tanto de acceso como económicos). Esto hace que para el estudiante sea compleja la elección de qué certificación le conviene tomar para la progresión efectiva de su carrera profesional. Portales especializados realizan anualmente listas de las certificaciones profesionales privadas con mayor aceptación por subárea de conocimiento dentro de la Informática.El portal tom's IT Pro (Tittle, 2015) haceunadivisión en 27 subáreas (Big Data, 
Linux, Programming, System Administrator, Information Security, Computer Networking, etc.)La mayoría de subáreas se cubren en mayor o menor medida en los títulos oficiales de Grado en Ingeniería Informática en España, aunque no con tanta profundidad ni concreción como en los cursos de preparación para las certificaciones profesionales privadas.

\section{Contenidos de Redes de Computadores en el Título de Graduado en Ingeniería Informática en la Universidad de Córdoba}

El título de Graduado en Ingeniería Informática en la Universidad de Córdoba se aprobó por Resolución Rectoral 10122, de 23 de mayo de 2011, de la Universidad de Córdoba, publicada en BOE (Resolución Rectoral 10122, 2011) y modificado en 2015, (Resolución Rectoral 3596, 2015). Dicho plan de estudio se basa en el documento VERIFICA (Universidad de Córdoba, 2015) aprobado por la Agencia Andaluza del Conocimiento en la que se indican las competencias que este título oficial otorga. Dicho título es impartido por la Escuela Politécnica Superior de Córdoba (Escuela Politécnica Superior de Córdoba, 2015).

Este título se presenta con tres menciones (o especialidades): Computación, Ingeniería de Computadores e Ingeniería del Software. Un estudiante que curse cualquiera de las tres menciones obtendrá el título de "Graduado en Ingeniería Informática", aunque en el Suplemento Europeo al Título se le indicará que tiene la correspondiente mención o especialidad. Dicha especialización se escoge por el estudiante al comenzar el $3^{\text {er }}$ curso de la titulación. El estudiante cursa un conjunto de asignaturas comunes para los tres perfiles de especialización (1 ${ }^{\text {er }}$ curso completo, $2^{\circ}$ curso completo, 4 asignaturas de $3^{\text {er }}$ curso y 1 asignatura de $4^{\circ}$ curso) y un grupo de asignaturas obligatorias de cada uno de los perfiles ( 6 asignaturas en 3er curso y 2 asignaturas de $4^{0}$ curso), además de un conjunto de créditos de formación de asignaturas optativas (30 créditos ECTS), que pueden ser escogidas entre optativas genéricas, optativas de cualquier perfil de especialización u otras opciones (prácticas en empresa, etc.)

Entre todas las competencias incluidas en el título, cabe destacar las siguientes competencias relacionadas con las Redes de Computadores:

- CEC11 (Competencia Específica Común): Conocimiento y aplicación de las características, funcionalidades y estructura de los Sistemas Distribuidos, las Redes de Computadores e Internet y diseñar e implementar aplicaciones basadas en ellas.

- CTEIC4 (Competencia de Tecnología Específica. Ingeniería de Computadores): Capacidad de diseñar e implementar software de sistema y de comunicaciones.

- CTEIC8: Capacidad para diseñar, desplegar, administrar y gestionar redes de computadores.

Estas competencias se proporcionan al cursar tres asignaturas del título: "Arquitectura de Redes", "Redes" y "Redes de Altas Prestaciones".

\subsection{Arquitectura de Redes}

Es una asignatura obligatoria de 6 créditos ECTS que se cursa en el $2^{\circ}$ cuatrimestre del $2^{\circ}$ curso de la titulación. Proporciona la primera aproximación a las Redes de Computadoras, centrándose principalmente en las capas inferiores del modelo de comunicaciones (nivel físico, nivel de enlace y nivel de red). En la descripción de contenidos del documento VERIFICA, aprobado por la Agencia de Evaluación Andaluza, se expone:

\section{- Breve descripción de contenidos.}


- Introducción y Conceptos Generales.

- Arquitectura de las redes de comunicación.

- Protocolos de las redes de comunicación.

- Transmisión de datos.

- Introducción a las Redes de área local y Redes de área extensa.

Como se puede observar la descripción es muy genérica, poco concreta, y deliberadamente flexible. Esto permite amoldar esta asignatura con facilidad a nuevas propuestas más concretas, que pueden fácilmente incorporarse dentro de cada uno de los contenidos del documento VERIFICA.

\subsection{Redes}

Esta asignatura (6 créditos ECTS) de 1er cuatrimestre de 3er curso es común a las tres especialidades. Se continúa la progresión conceptual y técnica de las Redes de Comunicaciones, tomando los niveles superiores del modelo de comunicaciones (nivel de red, nivel de transporte y nivel de aplicación). Los contenidos incluidos en el documento VERIFICA para esta asignatura son los siguientes:

- Introducción a las Redes de Computadores. Sistemas de comunicación y redes de computadoras, usos de las redes de computadores, modelo general de las comunicaciones, tipos de Redes de transmisión de datos, terminología y servicios, modelos de capas.

- La Capa de Red. Descripción general de la Capa de Red, redes de conmutación de circuitos, redes de conmutación de paquetes, algoritmos de enrutamiento, algoritmos de control de congestión, calidad de servicio en los sistemas de red.

- La capa de Red en Internet. Topología y arquitectura de Internet, direccionamiento en Internet, protocolo IP, protocolo de control ICMP, protocolos de encaminamiento en Internet (BGP, RIP, OSPF), transmisión multidestino (IGMP), IPv4 e IPv6.

- La Capa de Transporte. Descripción general de la capa de transporte, servicios proporcionados por la capa de transporte, elementos de los protocolos de transporte, programación de Sockets, protocolo UDP y TCP.

- La Capa de Aplicación. Descripción general de la capa de aplicación, modelo cliente/servidor, el sistema de nombres de dominio (DNS), correo electrónico, World Wide Web, Multimedia.

- Gestión y seguridad en Redes. Cifrado, autenticación y firma digital, certificados digitales, vulnerabilidades de TCP, protocolos IPSec y SSL-TLS, redes privadas virtuales, control de accesos en sistemas en red: firewall.

Esta descripción de contenidos es mucho más extensa y concreta que en la anterior, por lo que no hay tanta flexibilidad, aunque al ser un contenido tan amplio, prácticamente cualquier adición de nuevo contenido puede tener cabida en mayor o menor grado.

\subsection{Redes de Altas Prestaciones}

Dicha asignatura es obligatoria de la especialidad "Ingeniería de Computadores" y se cursa en el $1^{\text {er }}$ cuatrimestre del $4^{\circ}$ curso, con una carga de 6 créditos ECTS. Se centra en el estudio del diseño eficiente y configuración de Redes de Computadores para su optimización en términos de máximo rendimiento. 
Por su parte, el documento VERIFICA de este título, incluye los siguientes contenidos para esta asignatura:

- Redes de Área Local.

- Redes de Área Extensa

- Implementación Física de Dispositivos de Comunicaciones

- Interconexión de computadores paralelos

Estos contenidos son muy generales, por lo que presentan una gran flexibilidad para adaptar nuevos contenidos específicos dentro de cada uno de los bloques existentes.

En resumen, los estudiantes tienen 3 asignaturas propias de Redes de Computadores a lo largo de su titulación, que les proporcionan unas competencias específicas en dicho ámbito.

\section{CCNA: Certificación CISCO en Redes de Comunicación entre Ordenadores}

Una de las grandes empresas en el ámbito de las comunicaciones digitales es CISCO. Esta empresa no sólo construye productos hardware para comunicaciones (routers, switches, módems, etc.) sino que también colabora en el desarrollo protocolos y estándares de comunicación entre ordenadores. Todo esto ha hecho de esta gran multinacional, un referente de prestigio mundial en las comunicaciones entre ordenadores. Por último, aunque no menos importante, ha establecido un sistema de certificación de competencias, capacidades y conocimientos ligados a diversos aspectos de las Redes de Comunicación entre Ordenadores (en inglés, ComputerNetworking).

La empresa Cisco Systems posee un programa de certificaciones profesionales (CiscoSystems, 2015) que establece cinco niveles de certificación:

- Entry: Punto de inicio para personas que deseen comenzar su carrera como profesional de las redes de comunicaciones. Es un nivel muy básico, destinado para estudiantes que no posean ningún tipo de formación informática. Sin prerrequisitos de acceso.

- Associate: Presenta los fundamentos de las Redes de Computadores, y permite garantizar que los profesionales certificados son capaces de realizar la instalación, configuración, puesta en marcha y resolución de los problemas de las Redes de Computadores. Sin prerrequisitos de acceso.

- Professional: Muestra un nivel de certificación avanzado con habilidades y conocimientos sobre Redes de Computadores mucho más elevados. Requisito de acceso de certificación Associate.

- Expert: Está mundialmente reconocido como la certificación profesional privada más prestigiosa en Redes de Computadores. Requisito de acceso de certificación Professional.

- Architect: Es el nivel de certificación más elevado que se puede alcanzar y reconoce la máxima capacidad arquitectónica de diseño de Redes de Computadores en los entornos más complejos existentes. Requisito de acceso de certificación Expert.

Como se indica en la lista anterior, no existen prerrequisitos para optar a los niveles de certificación Entry o Associate, es decir, cualquier estudiante puede acceder a cualquiera de los cursos existentes en dichos niveles de certificación. Para el resto de niveles de certificación, sí existen algunos prerrequisitos de certificación previa. 
Como se ha indicado anteriormente, el nivel de certificación Entry no requiere conocimientos informáticos previos y por tanto, no proporciona conocimientos ni competencias adicionales que no se hayan adquirido en la Titulación oficial de Grado en Ingeniería Informática. Por tanto, dicho nivel de certificación tiene interés para estudiantes que cursen otras titulaciones y que deseen adquirir conocimientos certificados en el ámbito de las Redes de Computadores a un nivel elemental.Por tanto, el nivel de certificación Associatees el de principal interés para estudiantes y egresados del Grado en Ingeniería Informática, ya que no hay prerrequisitos adicionales y el nivel de conocimientos es similar (o ligeramente superior) al establecido en las asignaturas del título oficial de Grado en Ingeniería Informática.

Cisco Systems presenta 11 certificaciones diferentes de nivel Associate: CCDA ${ }^{2}, \mathrm{CCNA}^{3}$ Cloud, CCNA Collaboration, CCNA Data Center, CCNA Industrial, CCNA Security, CCNA ServiceProvider, CCNA Video, CCNA Voice, CCNA Wireless y CCNA Routing and Switching. De todos ellos, el más versátil y más demandado por las empresas es el CCNARouting and Switching.

\subsection{CertificaciónCCNA Routing and Switching}

Este curso pertenece al nivel de acreditación Associate y por lo tanto, se puede acceder de manera directa sin requisitos previos. Este certificado ha sido diseñado como un programa de tres años, aunque permite realizarse en un lapso de tiempo menor. Para obtener este certificado hay dos opciones:

- Aprobar el examen "200-120 CCNA" (Un examen de 50-60 preguntas con una duración total de 90 minutos).

- Aprobar dos exámenes "100-101 Interconnecting Cisco NetworkingDevices - Part 1 (ICND1) y "200-101 ICND2" (Cada examen tiene una duración de 90 minutosy consta de 50-60 preguntas).

Cisco Systems presenta una plataforma de e-learning para la formación de los aspirantes a estos certificados. El temario de este programa de certificación viene descrito en la Tabla 1, Tabla 2 y Tabla 3, para la preparación para los exámenes 200-120 CCNA, ICND1 e ICND2, respectivamente.

El certificado CCNA Routing and Switching tiene una validez de 3 años tras su obtención, tras lo cual se debe volver a recertificar, tomando de nuevo alguno de los exámenes que permiten revalidarlo.

Tabla 1. Contenidos del examen 200-120 CCNA del programa CCNA Routing and Switching

\begin{tabular}{|c|c|}
\hline RESPUESTA & Porcentajes (\%) \\
\hline Operación de Redes IP & $5 \%$ \\
\hline Tecnologías LAN Switch & $20 \%$ \\
\hline Direccionamiento IP (IPv4/IPv6) & $5 \%$ \\
\hline Tecnologías de Enrutamiento IP & $20 \%$ \\
\hline Servicios IP & $10 \%$ \\
\hline Seguridad en dispositivos de red & $10 \%$ \\
\hline
\end{tabular}

\footnotetext{
${ }^{2}$ Cisco Certified Design Associate.

${ }^{3}$ Cisco Certified Networking Associate.
} 


\begin{tabular}{|c|c|}
\hline Resolución de problemas & $20 \%$ \\
\hline Tecnologías WAN & $10 \%$ \\
\hline
\end{tabular}

Tabla 2. Contenidos del examen ICND1 del programa CCNA Routing and Switching

\begin{tabular}{|c|c|}
\hline RESPUESTA & Porcentajes (\%) \\
\hline Operación de Redes IP & $6 \%$ \\
\hline Tecnologías LAN Switch & $21 \%$ \\
\hline Direccionamiento IP (IPv4/IPv6) & $11 \%$ \\
\hline Tecnologías de Enrutamiento IP & $26 \%$ \\
\hline Servicios IP & $8 \%$ \\
\hline Seguridad en dispositivos de red & $15 \%$ \\
\hline Resolución de problemas & $13 \%$ \\
\hline
\end{tabular}

Tabla 3. Contenidos del examen ICND2 del programa CCNA Routing and Switching

\begin{tabular}{|c|c|}
\hline RESPUESTA & Porcentajes (\%) \\
\hline Tecnologías Switch LAN & $21 \%$ \\
\hline Tecnologías de Enrutamiento IP & $26 \%$ \\
\hline Servicios IP & $6 \%$ \\
\hline Resolución de Problemas & $32 \%$ \\
\hline Tecnologías WAN & $15 \%$ \\
\hline
\end{tabular}

Como puede observarse el examen 200-120 CCNA presenta todos los contenidos de los exámenes ICND1 e ICND2, variando los porcentajes. Este examen (200-120 CCNA) está principalmente recomendado a profesionales que deseen obtener la certificación en un periodo de tiempo menor a 3 años, con un mayor nivel de implicación en el autoestudio. La vía de certificación a través de dos exámenes es más progresiva aunque más lenta. El examen ICND1 permite conseguir la certificación CCENT (Nivel de acreditación Entry de Cisco Systems), por lo que aunque se realicen dos exámenes, con el primero se puede obtener una certificación y con el segundo otra certificación. Por tanto, si se posee la certificación Entrycon un periodo menor a 3 años, sólo es necesario realizar el examen ICND2 para alcanzar la certificación Associate, CCNA Routing and Switching.

Todos los exámenes tienen una parte de evaluación de conocimientos teóricos y otros de evaluación de conocimientos prácticos (bien a través de programas de ordenador que son simuladores de redes, o bien, mediante práctica en laboratorios reales utilizando diversos componentes físicos de redes).

\subsection{Currículum preparatorio del certificado CCNA Routing and Switching}

Para lograr alcanzar el certificado CCNA Routing and Switching, Cisco System propone una docencia estructurada en un programa de 4 cursos, denominado CCNA Curriculum. Dicho programa presenta la siguiente distribución: 
- CCNA1: Introducción a las Redes.Primer cursodel Currículum CCNA. Incluye la descripción de la arquitectura, la estructura, funcionalidades y componentes de Internet y de las Redes de Computadores, como la construcción de redes LAN sencillas, configuraciones básicas para routers y switches, e implementación de esquemas de direccionamiento IP.

- CCNA2: Fundamentos de Enrutamiento elntercambio de paquetes en Redes.Segundo curso del Currículum CCNA. En él se indica cómo configurar un router y un switch para proporcionar funcionalidad básica, gracias a la que se podrán configurar y resolver problemas comunes ligados a RIPv1, RIPng, OSPF monoárea y multiárea, VLANs y enrutamiento entre diferentes VLANs.

- CCNA3: Escalado de Redes.Este curso enseña a configurar routers y switches con funcionalidades más avanzadas, resolviendo problemas comunes con OSPF, EIGRP y STP.

- CCNA4: Conexión de Redes.En este último curso se cubren las tecnologías WAN y los servicios de red empleados para dar soporte a las aplicaciones dentro de redes complejas.

De manera adicional al aspecto teórico, Cisco Systems propone para cada curso un conjunto de prácticas en el laboratorio con sistemas de comunicaciones y materiales reales. En dichas prácticas, los alumnos deben interconectar componentes (cables, conectores, concentradores, routers, switches, etc.), configurarlos, comprobar el correcto funcionamiento, etc. Por otra parte, Cisco Systems ha desarrollado un conjunto de simuladores de gran precisión, entre los que destaca Cisco PacketTracer, en la que se pueden realizar todas las prácticas simuladas, para posteriormente poder implementarlas en el laboratorio.

La Gráfica 1 muestra la progresión de contenidos en el Currículum CCNA Routing and Switching y su correspondencia con las certificaciones obtenibles (CCENT y CCNA) según los contenidos estudiados.
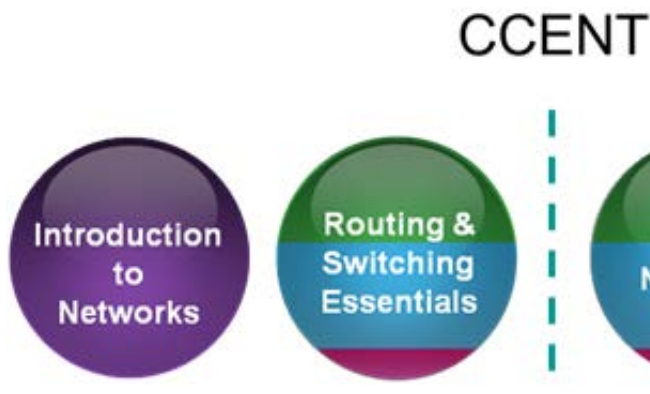

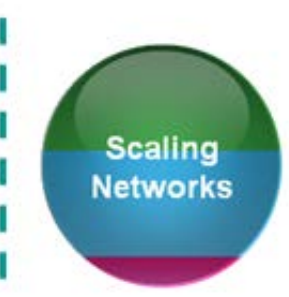

\section{CCNA}

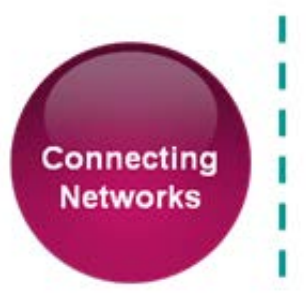

Gráfico 1. Progresión en el Currículum CCNA Routing and Switching.

\section{Adaptación de la certificación CCNA Routing and Switching al título de Graduado en Ingeniería Informática en la Universidad de Córdoba}

En 2010, un grupo de profesores de la Escuela Politécnica Superior de Córdoba (EPSC) de la Universidad de Córdoba se constituyó como Grupo de Trabajo en Redes y Seguridad como plataforma para el desarrollo de la docencia y la investigación en dichos ámbitos. La idea original era trabajar para que los alumnos no tuviesen que matricularse en otros centros para realizar los exámenes de certificación CCNA Routing and Switching/CCENT, sino que la propia Universidad de Córdoba pudiese proporcionar la certificación de dichos alumnos. 


\subsection{Academia Local Cisco: Córdoba Cisco NetworkingAcademy - Univ de Córdoba}

Para poder proporcionar la certificación CCNA Routing and Switching a los alumnos del título oficial, es necesario que la docencia se imparta por un conjunto de tutores/profesores con nivel de certificación de instructor (denominado $\mathrm{CCAI}^{4}$ ).

Para alcanzar esta certificación especial, se deben cumplir varias condiciones muy estrictas y exigentes, la primera es que es necesario trabajar para una institución considerada Academia Cisco. Por tanto, como tarea inicial el grupo de trabajo tomo la decisión de constituirse como Academia Local Cisco, que es el nivel más básico de Academia Cisco.

Para poder constituirse como Academia Local Cisco fue necesario cumplir un conjunto de requisitos, unos de tipo organizativo como obtener la tutela de una Academia Cisco de nivel Regional, que en este caso fue la de una Academia Regional Cisco, (regionalcit.com, 2015) sita en Cádiz, y otros de infraestructuras exigidas: instalar un laboratorio completo de Redes con múltiples dispositivos Cisco Systems, poseer ordenadores para los estudiantes y proporcionar conectividad a Internet.

Para ello, gracias a la ayuda de la EPSC, se adquirió el material de laboratorio exigido para poder impartir el certificado CCNA Routing and Switching. Por su parte, la Universidad de Córdoba cedió y acondicionó convenientemente un espacio para implantar dicho laboratorio.

Por último, se exigía al menos un profesor/tutor con certificación CCAl. En cuyo caso, uno de los profesores que impartía Redes de Computadores, poseía la certificación CCNA Routing and Switching y la certificación CCAI para dicho curso.

\subsection{Formación del Instructores}

Para poder proporcionar un equipo docente robusto y capaz, un total de 14 profesores de la EPSC tomaron la certificación CCAI para el curso CCNA1. Entre estos profesores se incluyeron todos los profesores que impartían la docencia de Redes de Computadores en el Grado de Ingeniería Informática y en las titulaciones oficiales a extinguir - I.T. Informática de Sistemas e Ingeniería en Informática, así como otros profesores interesados en apoyar e impulsar el proyecto de certificación CISCO.

El examen de certificación como Instructor requiere superar las pruebas del curso que se desea alcanzar nivel Instructor con un nivel del 80\% (en lugar del 55\% en el caso de certificación estándar).

El primer curso de implantación consiguieron la acreditación CCAI del curso CCNA1 los 14 profesores. El segundo curso de implantación, tan sólo 4 profesores tomaron la acreditación CCAI del curso CCNA2, al tener un nivel de exigencia más elevado y aumentar el encargo docente en titulaciones oficiales debido al Decreto-Ley 14/2012 (Decreto 14/2012, 2012). Tras este curso, dichos 4 profesores han conseguido las acreditaciones CCAI de los cursos CCNA3 y CCNA4. El resto de profesores ha indicado su intención de ir alcanzado las acreditaciones CCAI de los diferentes cursos de manera paulatina con una cadencia más tranquila.

\subsection{Implantación de la Academia Local: Córdoba Cisco NetworkingAcademy - Univ de Córdoba}

La implantación de la Academia Local Cisco se realizó gracias al apoyo económico e institucional tanto de la Escuela Politécnica Superior de Córdoba, como de la Universidad de Córdoba y del Consejo Social de la Universidad de Córdoba. A través de diversos planes propios de innovación docente, premios de innovación educativa y proyectos coordinados de mejora e

${ }^{4}$ CCAI: Cisco CertifiedAcademy Instructor 
innovación educativa de las titulaciones oficiales se ha logrado la implantación de esta propuesta.

La estructura de impartición de los contenidos se ha estructurado en dos vías cooperativas:

- Docencia oficial: Adaptando los contenidos teóricos y prácticos de las asignaturas del título oficial de Graduado en Ingeniería Informática.

- Docencia en los cursos del Currículum CCNA Routing and Switching: Se proporciona acceso a los laboratorios de informática para el acceso a la documentación electrónica para su estudio y para la realización de las pruebas de evaluación oficiales de cada curso.

Siguiendo esta estructura no se imparte docencia en exclusiva ligada a los cursos del Currículum CCNA Routing and Switching, sino que se utiliza la docencia obligatoria en la titulación oficial para proporcionar los conocimientos requeridos en las pruebas de evaluación de cada curso CCNA. El nivel de exigencia de la evaluación de cada asignatura del título oficial se mantiene tal y como se establece en el documento VERIFICA, aunque se tiende a equipararlo al de las pruebas de evaluación de los cursos CCNA.

\subsubsection{Plan Piloto de certificación Cisco}

En el curso 2011/2012, la Escuela Politécnica Superior de Córdoba de la Universidad de Córdoba convocó unas ayudas para Mejora de la Docencia. Se presentó un proyecto inicial para la adquisición del material de laboratorio Cisco, para la formación del profesorado como Instructor y para la implantación como Plan Piloto en las titulaciones oficiales de I.T. Informática de Sistemas y $2^{\circ}$ ciclo de Ingeniería en Informática. En este caso, no se contempló el Grado en Ingeniería en Informática ya que al ser una titulación de nueva implantación, aún no se había comenzado a impartir las asignaturas de Redes de Computadoras.

Se analizaron los contenidos de las asignaturas de Redes de Computadores y se observó que, para los alumnos que cursaran la titulación I.T. Informática de Sistemas y posteriormente $2^{\circ}$ ciclo de Ingeniería en Informática, ningún concepto de los exigidos en el curso CCNA1 se quedaba sin impartir en las diversas asignaturas relacionadas de dichas titulaciones.

Ese primer curso 2011-2012, como experiencia piloto, 3 alumnos tomaron el curso CCNA1, superando todos ellos las pruebas de certificación de dicho curso. Hay que indicar que la academia local puede expedir unos títulos (o certificados) por cada nivel superado del Currículum CCNA cuya validez profesional dependerá de la empresa que evalúe el currículum del alumno, aunque se suelen tener muy en cuenta en el entorno informático actual español.

A pesar del reducido número de alumnos que se incorporaron al plan piloto, los resultados, animaron a continuar la experiencia y a adaptar los contenidos de las asignaturas relacionados con Redes de Computadoras en el Grado en Ingeniería Informática para proporcionar todos los contenidos adaptados al Currículum CCNA Routing and Switching.

\subsubsection{Adaptación de las asignaturas del Grado en Ingeniería Informática}

Todos estos planes de mejora han ayudado a la adquisición del material, a la constitución de los planes piloto de implantación de la certificación CCNA Routing and Switching, a la formación del profesorado como Instructores, etc. Sin embargo, la principal innovación es el estudio de la adaptación de las asignaturas de Redes de Computadoras del título oficial de Grado en Ingeniería Informática al plan formativo del Currículum CCNA Routing and Switching.

Como hipótesis de partida, el grupo de trabajo en Redes y Seguridad Informática, impulsor de la implantación de la certificación en las ingenierías, considera que la certificación CCNA Routing 
and Switching y en particular, los contenidos preparatorios para el examen de certificación impartidos en los diferentes cursos del Currículum CCNA Routing and Switching pueden servir para una mejor adaptación de los contenidos y competencias específicas en las asignaturas relativas a Redes de Computadores en el título oficial de Graduado en Ingeniería Informática. Dicha adaptación de contenidos debe hacerse dentro del marco legal que obliga el documento VERIFICA acreditado por la ANECA para el título de Graduado en Ingeniería Informática de la Universidad de Córdoba.

Como primera aproximación, se realizó un estudio exhaustivo de los contenidos teóricos de todas las asignaturas relativas a Redes de Computadores de Graduado en Ingeniería Informática, comprobándose que todos los conceptos teóricos requeridos en la certificación CCENT se imparten. Sin embargo, sólo los estudiantes que cursen la asignatura de "Redes de Altas Prestaciones" (asignatura obligatoria en la Mención/Especialidad de Ingeniería de Computadores dentro del título de Graduado en Ingeniería Informática) podrán cubrir todos los conceptos teóricos requeridos para la certificación CCNA Routing and Switching. Para suplir el problema para estudiantes de otras Especialidades dentro de Graduado en Ingeniería Informática, se oferta la asignatura "Redes de Altas Prestaciones" como optativa para el resto de Menciones de la titulación. Se han encontrado pequeñas lagunas en aspectos muy concretos de carácter específico (protocolos y otros servicios de red) que no se cubrían con profundidad en los contenidos originales de las asignaturas involucradas en el título oficial, que se han incorporado en el temario, como extensiones, trabajos autónomos del alumnado, etc. para que no tuviese ningún tipo de interferencia con lo aprobado en el documento VERIFICA.

De manera colateral, se estableció la ruta de asignaturas del título oficial y su equivalencia con los diversos cursos del Currículum CCNA Routing and Switching para que el alumnado supiera la correspondencia entre las asignaturas cursadas y las de certificación. Como se puede observar, en este caso existe un problema de adaptación directa, ya que hay 3 asignaturas relativas a Redes de Computadores en el título oficial de Graduado en Ingeniería Informática en la Universidad de Córdoba (a saber, "Arquitectura de Redes", "Redes" y "Redes de Altas Prestaciones") mientras que el Currículum CCNA Routing and Switching está estructurado en 4 cursos. Por ello, todas las asignaturas del título oficial deben cubrir más de un curso del Currículum CCNA Routing and Switching. En la Tabla 4 se muestran dichas equivalencias. A su vez, se está trabajando para incorporar cursos de extensión universitaria, que de manera adicional, permita a los estudiantes profundizar y analizar con mayor detalle y detenimiento los diversos contenidos que puedan tener una mayor dificultad para los alumnos. Esto permitiría cubrir el contenido horario aconsejado en el Currículum CCNA Routing and Switching.

Tabla 4. Tabla de equivalencias entre Asignaturas cursadas en el título oficial de Graduado en Ingeniería Informática y el Currículum CCNA Routing and Switching

\begin{tabular}{|c|c|}
\hline Asignatura Oficial & Curso CCNA \\
\hline Arquitectura de Redes & CCNA1 \\
$\left(2^{\circ}\right.$ curso $)$ & CCNA2 \\
\hline Redes & CCNA1 \\
$\left(3^{\text {er }}\right.$ curso $)$ & CCNA2 \\
\hline Redes de Altas & CCNA3 \\
Prestaciones & CCNA4 \\
\hline
\end{tabular}


El principal escollo encontrado ha sido el aspecto práctico (tanto laboratorio como simulación), ya que las prácticas incluidas en el Currículum CCNA Routing and Switching hacen uso intensivo del material y los sistemas de Cisco System, mientras que las planteadas originalmente en las asignaturas de las titulaciones era de tipo general o de uso público. Sin embargo, la adaptación de las mismas prácticas utilizando el material y los recursos exigidos por Cisco System es directa y no interfiere en la planificación ni en la organización de las asignaturas. Además, en el documento VERIFICA de la titulación no se hace referencia a prácticas concretas en ninguna asignatura, por lo que, la adaptación de la propuesta original de las asignaturas del título oficial tan sólo ha requerido rehacer las guías docentes, lo que se adapta perfectamente al documento VERIFICA.

\section{Resultados del proceso de incorporación de la Certificación CCNA Routing and Switching al título de Graduado en Ingeniería Informática en la Universidad de Córdoba}

Tras 4 cursos tras la implantación inicial, se han podido observar una serie de resultados de tipo cuantitativo (número de matriculados y aprobados) como de tipo cualitativo. Se han analizado datos de los alumnos matriculados en el Currículum CCNA Routing and Switching en cada uno de los cuatro niveles (CCNA1, CCNA2, CCNA3 o CCNA4). El coste de dicha matrícula es gratuito, para evitar sobrecargar la economía de los estudiantes. Los estudiantes únicamente abonarían las tasas de examen de certificación en su caso, ya que éstas están fijadas por la propia Cisco Systems. Sin embargo, el procedimiento de certificación es totalmente externo a la Universidad de Córdoba, quedándose la Universidad de Córdoba como formadora de los cursos de preparación para los exámenes de certificación a través de la Academia Local Cisco, Córdoba Cisco NetworkingAcademy - Univ de Córdoba.

Hay que indicar que la matriculación de los estudiantes en el Curriculum CCNA Routing and Switching es voluntario, por lo tanto, hay un volumen de alumnos que cursan las asignaturas relativas a Redes de Computadores en el Grado en Ingeniería Informática en la Universidad de Córdoba que han decidido no tomar los cursos y exámenes del currículum CCNA Routing and Switching. Los dos motivos principales que alegan los estudiantes para no matricularse de estos cursos son sobrecarga horaria (exceso de carga docente por otras asignaturas) 0 desinterés en la obtención de la certificación por no adaptarse a su objetivos profesionales futuros.

En la Tabla 5 se muestran los resultados cuantitativos del impacto de la incorporación del proceso de certificación CCNA Routing and Switching al título oficial de Graduado en Ingeniería Informática de la Universidad de Córdoba, con los alumnos matriculados en cada curso académico

Tabla 5. Alumnos matriculados y aprobados en cada curso del Currículum CCNA Routing and Switching

\begin{tabular}{|c|c|c|c|c|c|c|c|c|c|}
\hline \multirow{2}{*}{$\begin{array}{c}\text { Curso } \\
\text { Académico }\end{array}$} & \multicolumn{2}{|c|}{ CCNA1 } & \multicolumn{2}{c|}{ CCNA2 } & \multicolumn{2}{c|}{ CCNA3 } & \multicolumn{2}{c|}{ CCNA4 } & $\begin{array}{c}\text { Porcentaje } \\
\text { Aprobados } \\
\text { Total }\end{array}$ \\
\cline { 2 - 10 } & Matric. & Aprob. & Matric. & Aprob. & Matric. & Aprob. & Matric. & Aprob. & \begin{tabular}{c} 
Totaly \\
\hline $2011-2012$
\end{tabular} \\
\hline 2 & 3 & - & - & - & - & - & - & $100 \%$ \\
\hline $2012-2013$ & 33 & 30 & - & - & - & - & - & - & $90,9 \%$ \\
\hline $2013-2014$ & 58 & 32 & 14 & 10 & 14 & 6 & - & - & $55,8 \%$ \\
\hline $2014-2015$ & 19 & 17 & 10 & 9 & 7 & 7 & - & - & $91,7 \%$ \\
\hline
\end{tabular}


El número de matriculados en el curso 2013-2014 sufrió un significativo aumento con respecto al curso anterior, aunque el porcentaje de aprobados bajó de manera muy marcada. Esto fue debido a los resultados del curso académico anterior, que fueron muy buenos, lo cual hizo pensar a los estudiantes del siguiente curso académico que iban a poder superar las pruebas de evaluación del nivel CCNA1 con muy poco estudio, tal y como informaron muchos de los estudiantes suspensos al acabar dicho curso. De hecho, se puede comprobar que el volumen de suspensos más significativo se da en el curso CCNA1, con un índice de aprobados del $55.2 \%$.

Se ha observado un descenso en el curso 2014-15 en el número de matriculados por el traslado del laboratorio Cisco a una nueva ubicación, que provocó que se iniciaran las sesiones prácticas, junto con los exámenes de evaluación, con mucha posterioridad, teniendo que concentrarse todos ellos en un único cuatrimestre en lugar de en el curso completo. Ello desanimó a un grupo significativo de alumnos, que aunque mostraron interés en tomar los cursos, excedían la capacidad horaria de docencia semanal y no quisieron tomar el curso para no sobrecargarse en demasía.

Hay que indicar que en los datos de matriculación expuestos en la Tabla 5, se incluyen tanto alumnos que cursan $3^{\circ} 04^{\circ}$ de Graduado en Ingeniería Informática, como alumnos de $2^{\circ}$ ciclo de Ingeniería en Informática (título a extinguir). Es por ello, que en varios años el número de matriculados en los cursos del Currículum CCNA Routing and Switching fue superior al número de alumnos matriculados en las asignaturas de Redes de Computadores del Grado en Ingeniería Informática. Es de esperar que esta situación se normalice en los próximos cursos académicos al haberse finalizado la docencia en el título de $2^{\circ}$ ciclo de Ingeniería en Informática.

A nivel cualitativo, se han realizado consultas personalizadas a diversos estudiantes matriculados en alguno de los cursos del Currículum CCNA Routing and Switching para tener feedback de si la experiencia estaba siendo satisfactoria. Ante las respuestas proporcionadas, se han ido realizando los ajustes pertinentes para la mejora de la calidad de la docencia (tanto en la impartición de los cursos del Currículum CCNA Routing and Switching como en la impartición de las diversas asignaturas de Redes de Computadores en el Grado en Ingeniería Informática).

No se ha optado por utilizar un cuestionario sistematizado, ya que en las primeras fases de implantación de esta propuesta, se ha considerado más relevante los comentarios libres frente a las respuestas cerradas que presentan los cuestionarios tradicionales. Además, se ha considerado que los resultados para el curso 2014-15 podrían estar altamente sesgados por la influencia negativa del retraso en el inicio de los cursos por el traslado del laboratorio Cisco referido con anterioridad.

\section{Conclusiones}

Los resultados obtenidos muestran un claro interés por parte de los estudiantes de Graduado en Ingeniería Informática en la Universidad de Córdoba en la obtención del certificado CCNA. El profesorado implicado ha desarrollado una labor de adaptación del temario y contenidos teóricos y prácticos para conseguir que los estudiantes, que tienen que cursar de manera obligatoria las asignaturas del título oficial, obtengan las competencias específicas requeridas para lograr superar las pruebas de evaluación de cada curso del Currículum CCNA Routing and Switching. Además, todo ello sin coste económico añadido a los estudiantes.

Esta experiencia muestra que las certificaciones profesionales privadas pueden servir como guía para coordinar asignaturas de los títulos oficiales. Estas asignaturas oficiales reorganizarían los temarios, realizando las adaptaciones necesarias para proporcionar los contenidos requeridos por las certificaciones profesionales privadas. Estas modificaciones se ven limitadas 
por lo indicado en los documentos VERIFICA de los títulos oficiales, que restringen en gran medida las posibilidades de realizar alteraciones sustanciales en los contenidos.

Otra herramienta docente disponible son los cursos de extensión universitaria para reforzar aquellos aspectos que pudieran haber quedado con algún tipo de déficit en las asignaturas del título oficial. En este aspecto, hay que tener en cuenta lo que cada certificación pueda establecer en relación a dichos cursos en materia de limitación económica (si deben ser gratuitos, deben ser ofertados como seminarios u otras figuras de extensión universitaria exentas de pago de tasas) o de cualquier otro tipo (limitación mínima o máxima de docencia, uso permitido/exigido de material específico, etc.) La legislación universitaria en materia de docencia es suficientemente flexible como para poder encontrar soluciones viables, satisfactorias tanto para los estudiantes como para el profesorado, cumpliendo las exigencias legales que pudiera imponer la empresa certificadora.

Se ha de indicar que esta experiencia está teniendo muy buena acogida dentro de la Escuela Politécnica Superior de Córdoba, especialmente entre el alumnado y se ha planteado la posibilidad de extender esta experiencia a otras facultades que pudieran estar interesadas en ofertar a sus estudiantes contenidos que les permitiesen optar a algún tipo de certificación dentro del ámbito informático, como a otras certificaciones informáticas de otros campos: Certificación en Linux, Certificación en Java/Android, Certificación Microsoft, etc. Es de suponer que, en caso de que se decida incluir dichas certificaciones como beneficio colateral de las titulaciones oficiales, se utilizará la propuesta descrita en este trabajo para su posible implantación práctica de las mismas dentro de las asignaturas de los títulos oficiales involucrados.

\section{Agradecimientos}

Este laboratorio ha sido dotado económicamente por la Escuela Politécnica Superior de Córdoba y la Universidad de Córdoba a través de proyectos de innovación docente asociados al Espacio Europeo de Educación Superior, y a través de proyectos de innovación educativa asociados a los títulos de Graduado en Ingeniería Informática, cediéndose también el espacio para el laboratorio en el aulario Averroes. También ha colaborado en la financiación del proyecto el Consejo Social de la UCO.

Se quiere agradecer a todos los profesores, colaboradores y alumnos del Grupo de Trabajo de Redes y Seguridad Informática de la Universidad de Córdoba su implicación, dedicación, esfuerzo y entusiasmo con el que han conseguido que esta experiencia haya alcanzado este nivel.

\section{Referencias}

ANECA. (2005). Libro Blanco. Título de Grado en Ingeniería Informática. Agencia Nacional de Evaluación de la Calidad y Acreditación. (Spain). $<$ http://www.aneca.es/media/150388/libroblanco jun05 informatica.pdf> [Consulta: 18 de agosto de 2015]

Cisco Systems (2015). Certifications - IT Certification and Career Paths - Cisco Systems.<http://www.cisco.com/web/learning/certifications/index.html> [Consulta: 19 de agosto de 2015]

Escuela Politécnica Superior de Córdoba (2015) Título de Graduado en Ingeniería Informática.<http://www.uco.es/eps/node/619>[Consulta: 19 de agosto de 2015] 
España. Real Decreto 517/2015, de 19 de junio, por el que se aprueban los Estatutos Generales de los Colegios Oficiales de Ingeniería Técnica en Informática y de su Consejo General. Boletín Oficial del Estado, 11 de julio de 2015,n.165, pp. 57792-57824.

España. Real Decreto 518/2015, de 19 de junio, por el que se aprueban los Estatutos Generales de los Colegios Oficiales de Ingeniería en Informática y de su Consejo General. Boletín Oficial del Estado, 11 de julio de 2015, n. 165, pp. 57825-57855.

España. Real Decreto-Ley 14/2012, de 20 de abril, de medidas urgentes de racionalización del gasto público en el ámbito educativo. Boletín Oficial del Estado, 21 de abril de 2012, n. 96, pp. 30977-30984.

España. Resolución Rectoral 10122, de 23 de mayo de 2011, de la Universidad de Córdoba, por la que se publica el plan de estudios de Graduado en Ingeniería Informática. Boletín Oficial del Estado, 10 de junio de 2011, n. 138, pp. 60025-60029.

España. Resolución Rectoral 3596, de 17 de marzo de 2015, de la Universidad de Córdoba, por la que se publica la modificación del plan de estudios de Graduado en Ingeniería Informática. Boletín Oficial del Estado, 2 de abril de 2015, n. 79, pp. 28109-28113.

Infojobs.net (2015) Portal de Empleos en España. <http://www.infojobs.net>[Consulta: 18 de Agosto de 2015]

Merle, V. (1997). La evolución de los sistemas de validación y certificación ¿Qué modelos son posibles y qué desafíos afronta el país francés? Revista europea de formación profesional, 12, pp. 39-52.

Regionalcit.es (2015) Cátedra RELEC - Centro Cisco de Soporte / Centro Cisco de Formación de Instructores <http://www.regionalcit.es/> [Consulta: 20 de agosto de 2015]

Ticjob.es (2015) Portal de empleos TIC en España. <http://www.ticjob.es/>[Consulta: 18 de agosto de 2015]

Tittel, E. (2015) Best IT Certifications for 2015. tom's IT Pro - Real-World Business Technology. <http://www.tomsitpro.com/articles/best-it-certifications,1-1352.html> [Consulta: 18 de agosto de 2015]

Universidad de Córdoba. (2015) Graduado en Ingeniería Informática por la Universidad de Córdoba.

$<$ http://www.uco.es/grados/index.php?option=com content\&view=article\&id=87:memoriade-ingenieria-informatica\&catid=8>[Consulta: 19 de agosto de 2015] 